\title{
Amplified Fragment Length Polymorphism Markers Linked to a Major Quantitative Trait Locus Controlling Scab Resistance in Wheat
}

\author{
Guihua Bai, Frederic L. Kolb, Gregory Shaner, and Leslie L. Domier
}

First author: NCAUR-USDA-ARS, 1815 North University Street, Peoria, IL 61604; second author: Department of Crop Sciences, University of Illinois, Urbana 61801; third author: Department of Botany and Plant Pathology, Purdue University, West Lafayette, IN 479071155; and fourth author: USDA-ARS, Urbana, IL 61801. Accepted for publication 29 January 1999.

\section{ABSTRACT}

Bai, G.-H., Kolb, F. L., Shaner, G., and Domier, L. L. 1999. Amplified fragment length polymorphism markers linked to a major quantitative trait locus controlling scab resistance in wheat. Phytopathology 89:343-348.

Scab is a destructive disease of wheat. To accelerate development of scab-resistant wheat cultivars, molecular markers linked to scab resistance genes have been identified by using recombinant inbred lines (RILs) derived by single-seed descent from a cross between the resistant wheat cultivar Ning 7840 (resistant to spread of scab within the spike) and the susceptible cultivar Clark. In the greenhouse, $\mathrm{F}_{5}, \mathrm{~F}_{6}, \mathrm{~F}_{7}$, and $\mathrm{F}_{10}$ families were evaluated for resistance to spread of scab within a spike by injecting about 1,000 conidiospores of Fusarium graminearum into a central spikelet. Inoculated plants were kept in moist chambers for 3 days to promote initial infection and then transferred to greenhouse benches. Scab symptoms were evaluated four times $(3,9,15$, and 21 days after inoculation). The frequency distribution of scab severity indicated that resistance to spread of scab within a spike was controlled by a few major genes. DNA was isolated from both parents and $\mathrm{F}_{9}$ plants of the 133 RILs. A total of 300 combinations of amplified fragment length polymorphism (AFLP) primers were screened for polymorphisms using bulked segregant analysis. Twenty pairs of primers revealed at least one polymorphic band between the two contrasting bulks. The segregation of each of these bands was evaluated in the 133 RILs. Eleven AFLP markers showed significant association with scab resistance, and an individual marker explained up to $53 \%$ of the total variation $\left(R^{2}\right)$. The markers with high $R^{2}$ values mapped to a single linkage group. By interval analysis, one major quantitative trait locus for scab resistance explaining up to $60 \%$ of the genetic variation for scab resistance was identified. Some of the AFLP markers may be useful in marker-assisted breeding to improve resistance to scab in wheat.
Wheat scab, also called Fusarium head blight, caused mainly by Fusarium graminearum Schwabe, is a destructive disease of wheat worldwide (4). Scab significantly reduces wheat grain yield and quality. The mycotoxins produced by $F$. graminearum in infected grains are detrimental to livestock and are also a safety concern in human foods. The use of resistant cultivars is an effective way to control the disease. Breeding for scab resistance with traditional methods requires substantial time and effort, because resistance is quantitatively controlled and because evaluation of resistance requires a misting system and laborious inoculation and evaluation procedures.

Molecular markers are powerful tools that can be used for marker-assisted selection (10) and as landmarks for map-based cloning of resistance genes (19). Molecular markers associated with quantitative trait loci (QTL) have been reported for many crops and many important traits $(9,16,17,26)$. After a linkage between a QTL and molecular markers has been determined, the QTL can be transferred into different genetic backgrounds by marker-assisted selection. Restriction fragment length polymorphism (RFLP) markers have been used successfully to analyze single gene traits and QTL $(9,17,26,32)$. Many RFLP markers for disease and insect resistance have been identified in wheat $(2,16,32)$, but because of the low level of polymorphism among wheat cultivars (6), it is difficult to detect polymorphisms in new wheat mapping populations with some RFLP probes. Recently, the application of polymerase chain reaction (PCR)-based molecular markers, especially

Corresponding author: F. L. Kolb; E-mail address: f-kolb@uiuc.edu

Publication no. P-1999-0301-03R

This article is in the public domain and not copyrightable. It may be freely reprinted with customary crediting of the source. The American Phytopathological Society, 1999. amplified fragment length polymorphisms (AFLPs), has provided powerful tools for the detection of a larger number of DNA polymorphisms in plants $(1,11,24,29,31)$.

In wheat, RFLPs and random amplified polymorphic DNAs (RAPDs) have been used to study wheat scab, and several markers were identified for scab resistance QTL $(3,21)$; however, all of these markers accounted for only a small portion of the variation. A low level of polymorphism of RAPDs and RFLPs within wheat may be a barrier to the identification of markers that are closely linked to major scab resistance genes $(3,6)$. AFLPs have the potential to generate a large number of polymorphic loci $(23,31)$. AFLPs combine the merits of both RFLP and PCR-based multilocus markers. Detection of a virtually unlimited number of restriction fragments in complex genomes is especially useful for plant species with low polymorphism. In other plant species, it has been shown that AFLPs are efficient for high resolution mapping and chromosome landing $(8,29)$.

Two major types of host plant resistance to $F$. graminearum are resistance to initial infection (type I) and resistance to the spread of $F$. graminearum within a spike (type II) (18). Resistance to initial infection by the fungus has not been well characterized, because it is highly influenced by environmental conditions. However, resistance to fungal spread is a rather stable measure of cultivar resistance, and the single floret inoculation method can be used to evaluate this type of resistance (4). When a single floret of a spike is inoculated with conidiospores, genetic differences in resistance to spread within the spike can be detected among wheat cultivars as a continuum ranging from highly resistant $(5 \%$ scabbed spikelets) to highly susceptible (up to $100 \%$ scabbed spikelets) (5). Only resistance to spread within a spike was investigated in this study.

The inheritance of resistance to spread of scab within a spike has not been well defined. Yu (34) and Chen (7) reported polygenic control of resistance to spread of scab in a spike, while more 
recent reports demonstrated that a few genes with major effects might be involved in resistance to spread of scab in a spike $(3,30)$. Estimates of the number of genes controlling scab resistance may be affected by the control of environmental variability. Molecular marker technology may provide new tools for further investigation of inheritance of wheat resistance to scab. Our objectives in this study were to characterize a major QTL for wheat resistance to spread of scab in a spike, identify AFLP markers associated with the QTL, and explore the potential of marker-assisted selection in improving wheat resistance to scab.

\section{MATERIALS AND METHODS}

Plant materials. Ning 7840 is a scab-resistant wheat cultivar from China derived from the cross ABPOPA/Anhui 11//Sumai 3. It is highly resistant to spread of scab within an inoculated spike. Clark (22) is a soft red winter wheat cultivar released by Purdue University, West Lafayette, IN. 'Clark' is extremely susceptible to spread of scab within spikes. A cross was made between 'Ning 7840' and 'Clark' during the fall of 1990, and 133 recombinant inbred lines (RILs) were derived by single-seed descent in a greenhouse at Purdue University. Leaf tissue from $\mathrm{F}_{9}$ plants grown in a greenhouse at the University of Illinois, Urbana-Champaign, was used for DNA isolation.

Scab evaluation. $F_{5}$ to $F_{7}$ generations of the RILs were evaluated for spread of scab within a spike in the greenhouse of Purdue University in 1994 and 1995, and $F_{10}$ plants were evaluated in the greenhouse at the University of Illinois in 1998. The inoculum of $F$. graminearum was a mixture of field isolates that originated from 10 randomly selected scabbed seeds of the susceptible cultivar Caldwell grown at the Purdue Agricultural Research Center in 1986. Conidiospores were produced in mung bean liquid medium as described by Bai and Shaner (5). For each experiment, RILs and parents were randomly arranged in greenhouse benches. The number of plants of each RIL inoculated in each generation varied from 9 to 16. In each generation, individual plants served as subsamples. RILs varied in flowering date. Plants were inoculated when a spike was just beginning to flower. Thus, by necessity, plants of different lines were inoculated on different dates. The inoculation date for each spike was recorded and used to determine when to collect data from that plant. Spikes were inoculated using a hypodermic syringe to inject about 1,000 conidiospores of F. graminearum into a central floret. Inoculated plants were placed in moist chambers and misted with tap water. The chambers were covered with polyethylene sheeting and maintained at 23 to $25^{\circ} \mathrm{C}$ with $100 \%$ relative humidity. Three days after inoculation, the plants were removed from the moist chambers and returned to the greenhouse benches. Greenhouse temperature averaged $23^{\circ} \mathrm{C}$ during the day with a range of 19 to $30^{\circ} \mathrm{C}$ and $19^{\circ} \mathrm{C}$ at night with a range of 17 to $21^{\circ} \mathrm{C}$.

Visual symptoms ranged from dark brown, water-soaked spots on the glumes to bleached spikelets. All of these symptoms were

TABLE 1. Phenotypic correlation coefficients for two scab severity measurements ${ }^{\mathrm{a}}$ assessed in four generations

\begin{tabular}{|c|c|c|c|c|c|c|c|}
\hline & \multicolumn{4}{|c|}{ AUDPC } & \multicolumn{3}{|c|}{ PSS } \\
\hline & $\mathrm{F}_{5}$ & $\mathrm{~F}_{6}$ & $\mathrm{~F}_{7}$ & $\mathrm{~F}_{10}$ & $\mathrm{~F}_{5}$ & $\mathrm{~F}_{6}$ & $\mathrm{~F}_{7}$ \\
\hline \multicolumn{8}{|c|}{ AUDPC } \\
\hline $\mathrm{F}_{6}$ & 0.65 & & & & & & \\
\hline $\mathrm{F}_{7}$ & 0.74 & 0.67 & & & & & \\
\hline $\mathrm{F}_{10}$ & 0.66 & 0.64 & 0.72 & & & & \\
\hline \multicolumn{8}{|l|}{ PSS } \\
\hline $\mathrm{F}_{5}$ & 0.97 & 0.63 & 0.73 & 0.64 & & & \\
\hline $\mathrm{F}_{6}$ & 0.63 & 0.92 & 0.66 & 0.62 & 0.63 & & \\
\hline $\mathrm{F}_{7}$ & 0.75 & 0.67 & 0.95 & 0.72 & 0.75 & 0.70 & \\
\hline $\mathrm{F}_{10}$ & 0.66 & 0.66 & 0.72 & 0.95 & 0.66 & 0.66 & 0.75 \\
\hline
\end{tabular}

a Area under disease progress curve (AUDPC) and percentage of scabbed spikelets (PSS) 21 days after inoculation. recorded as scabbed spikelets. Scabbed spikelets were counted 3, 9,15 , and 21 days after inoculation. Final disease severity values were calculated as the percentage of scabbed spikelets (PSS) per spike on the twenty-first day after inoculation. Also, the area under the disease progress curve (AUDPC) was calculated for each inoculated spike according to Shaner and Finney (27). Associations between markers and PSS were made with both log transformation and untransformed data in the QTL analysis. Since the nature of the association did not change substantially, the results with untransformed data are presented.

DNA preparation and bulked segregant analysis. Wheat leaves were harvested from $\mathrm{F}_{9}$ plants 3 weeks after seed germination. DNA was isolated from both parents ('Ning 7840' and 'Clark') and the $133 \mathrm{~F}_{9}$ RILs using the cetyltrimethylammonium bromide procedure (25). Based on greenhouse phenotypic evaluations from $F_{5}$ to $F_{7}$ generations, a DNA bulk was formed by pooling DNA from five scab-resistant RILs. A second DNA bulk was formed from five scab-susceptible RILs. The two parents and two bulks were screened for polymorphisms with all 300 AFLP primer combinations. Primer combinations showing AFLP products present in one bulk, but absent from the other, were used to evaluate all 133 RILs.

AFLP analysis. The AFLP analysis was conducted as described by Vos et al. (31) with the following modifications. For each sample, $500 \mathrm{ng}$ of genomic DNA was digested simultaneously with $E c o$ RI and MseI restriction enzymes and ligated to adapters (EcoRI and MseI adapters) with the following sequences: 5' CTCGTAGACTGCGTACC and 5' AATTGGTACGCAGTC for EcoRI; and 5' GACGATGAGTCCTGAG and 5' TACTCAAGGACTCAT for MseI.

Neither of the adapters was biotinylated, and the selection step using streptavidin-coated magnetic beads was omitted. PCR was performed in two consecutive reactions in a thermocycler (MJ Research, Inc., Watertown, MA). Primers were synthesized by Life Technologies, Inc., Gaithersburg, MD. The ligated DNA fragments were preamplified using a pair of primers as described by Vos et al. (31) without any additional selective nucleotide at the $3^{\prime}$ end. The sequences of the primers EP1 and MP1 were 5' GACTGCGTACCAATTC and 5' GATGAGTCCTGAGTAA, respectively.

Preamplification was performed in a total volume of $40 \mu$ containing $60 \mathrm{ng}$ each of primers EP1 and MP1, $0.2 \mathrm{mM}$ of all four dNTPs (Amersham Pharmacia Biotech, Piscataway, NJ), 1× PCR buffer (Life Technologies, Inc.), $1.75 \mathrm{mM}$ of $\mathrm{MgCl}_{2}, 1 \mathrm{U}$ of Taq DNA polymerase (Life Technologies, Inc.), and $10 \mathrm{ng}$ of ligated DNA fragments. The PCR amplification used the following parameters: 30 cycles of $94^{\circ} \mathrm{C}$ for $30 \mathrm{~s}, 56^{\circ} \mathrm{C}$ for $30 \mathrm{~s}$, and $72^{\circ} \mathrm{C}$ for $60 \mathrm{~s}$. The PCR products of the preamplification were diluted 1:40 and used

TABLE 2. Effect of allelic substitution for 11 amplified fragment length polymorphism loci based on area under disease progress curves (AUDPC) and percentage of scabbed spikelets (PPS) at 21 days after inoculation for $F_{7}$ and $F_{10}$ plants

\begin{tabular}{lccccc}
\hline & \multicolumn{2}{c}{ PSS } & & \multicolumn{2}{c}{ AUDPC } \\
\cline { 2 - 3 } \cline { 5 - 6 } Marker $^{\mathrm{a}}$ & $\mathrm{F}_{7}$ & $\mathrm{~F}_{10}$ & & $\mathrm{~F}_{7}$ & $\mathrm{~F}_{10}$ \\
\hline GCTG/CTC2 & $0.28^{\mathrm{b}}$ & 0.26 & & 3.2 & 2.8 \\
AAC/CTG7 & 0.28 & 0.26 & & 3.4 & 2.9 \\
AAG/AGC10 & 0.38 & 0.33 & & 4.5 & 3.5 \\
AAC/CGAC3 & 0.37 & 0.39 & & 4.2 & 4.0 \\
ACT/CAT3 & 0.33 & 0.35 & & 4.0 & 3.8 \\
GCTG/CGAC1 & 0.43 & 0.44 & & 4.8 & 4.4 \\
ACT/TGC7 & 0.41 & 0.43 & & 4.7 & 4.4 \\
CTCG/CTC9 & 0.37 & 0.37 & & 4.3 & 3.8 \\
GTG/CAGT10 & 0.38 & 0.41 & & 4.3 & 4.0 \\
AGT/CAGT8 & 0.38 & 0.40 & & 4.1 & 4.1 \\
CTCG/AGC1 & 0.43 & 0.41 & 4.8 & 4.0 \\
\hline
\end{tabular}

a Marker name consists of abbreviations of selective nucleotides the of primers EcoRI (left) and MseI (right) separated by a slash.

b All values are the differences between the two allelic groups, and they are significant at the 0.001 probability level in the analysis of variance. 
as templates for the selective amplification with a combination of two AFLP primers, each containing three or four selective nucleotides at the $3^{\prime}$ end. Primers used for selective amplification were similar to those described by Vos et al. (31) with the following extensions: AAC, AAG, ACA, ACAG, ACT, ACTG, AGC, ACC, ACG, AGG, AGT, GAC, GTG, GCTG, and CTCG for EcoRI; and CAC, CAG, CAGT, CAT, CTG, CTGA, CTT, CAA, CTA, AGC, ACC, TGC, GCG, GTG, CTC, GAC, CACG, CGAC, GCAG, and ACGC for $M s e I$.

The EcoRI selective primers were labeled with ${ }^{33} \mathrm{P}-\gamma \mathrm{ATP}$ (Andotek, Irvine, CA) followed by a second amplification reaction with 36 cycles as described by Vos et al. (31). The second amplifications were carried out in a total volume of $12 \mu \mathrm{l}$ containing $3.3 \mathrm{ng}$ of labeled EcoRI primer, $20 \mathrm{ng}$ of $M s e \mathrm{I}$ primer, $0.2 \mathrm{mM}$ of all four dNTPs, $1 \times$ PCR buffer, $0.4 \mathrm{U}$ of Taq DNA polymerase, and $3 \mu \mathrm{l}$ of template DNA. The PCR products were separated on $5 \%$ denaturing polyacrylamide gels at $100 \mathrm{~W}$. After electrophoresis, the gels were transferred to Whatman 3MM paper (Whatman International, Ltd., Maidstone, England), dried without prior fixation, and exposed to X-ray film (Bio-Max MR; Eastman Kodak Co., Rochester, NY) for about $48 \mathrm{~h}$. The AFLP bands were scored by visual inspection, and AFLP loci were named based on the combination of selective nucleotides in the selective primers (EcoRI and $M s e \mathrm{I})$ and size of a band. For example, ACG/GTG5 refers to the fifth polymorphic band (bands numbered from high to low molecular weight) amplified with the primer combination of EcoRI-ACG and MseI-GTG.

Marker analysis. Segregating AFLP markers were scored for each RIL as either 'Ning 7840'-type (A) or 'Clark'-type (B). Linkage analysis was performed by using the MAPMAKER program (version 2.0 for MacIntosh) (13) with the Kosambi mapping function (12). The linkage map was obtained by choosing 0.4 as the maximum recombination fraction and 6 as the minimum logarithm of the odds ratio (LOD) score value. For QTL analysis, singlefactor analysis of variance (ANOVA) and simple regression analysis (28) were performed using Microsoft Excel (Microsoft Corp., Redmond, WA). Broad-sense heritability was calculated based on single-factor ANOVA.

Interval mapping was conducted with MAPMAKER/QTL 1.1 (13). A LOD score threshold of 3.0 was used to declare the presence of a putative QTL in a given genomic region. To identify the putative chromosome location of the QTL, primer combinations that produced markers closely linked to the QTL were used to amplify DNA of RILs from the W7984/Opata M 85 cross (ITMI [International Triticeae Mapping Initiative] mapping population). The ITMI map data were retrieved from the online GrainGenes website maintained by the USDA, ARS National Agricultural Library. If a marker linked to the QTL was mapped in both populations, the

TABLE 3. $R^{2}$ values from simple regression analysis for 11 amplified fragment length polymorphism markers evaluated in four generations

\begin{tabular}{|c|c|c|c|c|c|c|c|c|}
\hline \multirow[b]{2}{*}{ Marker $^{\mathrm{a}}$} & \multicolumn{2}{|r|}{$\mathrm{F}_{5}$} & \multicolumn{2}{|r|}{$\mathrm{F}_{6}$} & \multicolumn{2}{|r|}{$\mathrm{F}_{7}$} & \multicolumn{2}{|c|}{$\mathrm{F}_{10}$} \\
\hline & $\mathrm{PSS}^{\mathrm{b}}$ & $\mathrm{AUDPC}^{\mathrm{c}}$ & PSS & AUDPC & PSS & AUDPC & PSS & AUDPC \\
\hline GCTG/CTC2 & 0.12 & 0.14 & 0.13 & 0.11 & 0.23 & 0.23 & 0.17 & 0.20 \\
\hline AAC/CTG7 & 0.13 & 0.14 & 0.12 & 0.11 & 0.22 & 0.23 & 0.16 & 0.21 \\
\hline AAG/AGC10 & 0.25 & 0.25 & 0.23 & 0.20 & 0.40 & 0.41 & 0.28 & 0.30 \\
\hline CTCG/CTC9 & 0.21 & 0.22 & 0.17 & 0.14 & 0.39 & 0.39 & 0.35 & 0.37 \\
\hline АCT/CAT3 & 0.16 & 0.18 & 0.11 & 0.10 & 0.31 & 0.33 & 0.31 & 0.35 \\
\hline GCTG/CGAC1 & 0.32 & 0.31 & 0.23 & 0.20 & 0.53 & 0.50 & 0.51 & 0.49 \\
\hline ACT/TGC7 & 0.29 & 0.27 & 0.24 & 0.20 & 0.50 & 0.47 & 0.48 & 0.48 \\
\hline CTCG/AGC1 & 0.32 & 0.29 & 0.27 & 0.20 & 0.52 & 0.47 & 0.39 & 0.38 \\
\hline AAC/CGAC3 & 0.22 & 0.19 & 0.27 & 0.24 & 0.40 & 0.36 & 0.40 & 0.39 \\
\hline AGT/CAGT8 & 0.25 & 0.23 & 0.28 & 0.24 & 0.43 & 0.37 & 0.42 & 0.42 \\
\hline GTG/CAGT10 & 0.25 & 0.23 & 0.30 & 0.25 & 0.45 & 0.40 & 0.43 & 0.42 \\
\hline
\end{tabular}

a Marker name consists of abbreviations of selective nucleotides from the primers EcoRI (left) and MseI (right) separated by a slash.

${ }^{\mathrm{b}}$ PSS $=$ Percentage of scabbed spikelets at 21 days after inoculation.

c AUDPC = Area under disease progress curve calculated from percentage of scabbed spikelets at $3,9,15$, and 21 days after inoculation. chromosome location of the marker in the ITMI map was tentatively considered as the chromosome location for the marker.

\section{RESULTS}

Scab resistance in RILs. Significant differences in both PSS and AUDPC were observed between the two parents in all four greenhouse evaluations. AUDPC in 1998 was $0.83 \pm 0.07$ for 'Ning 7840' and $10.7 \pm 0.38$ for 'Clark'. The mean broad-sense heritability for resistance to spread of scab in a spike was high (0.86) and ranged from 0.79 to 0.91 for both AUDPC and PSS in the four greenhouse tests (data not shown). The correlation coefficients between AUDPC and PSS were highly significant and ranged from 0.92 to 0.97 within generations for the four greenhouse evaluations (Table 1). Correlations for PSS or AUDPC among different greenhouse tests were also highly significant (Table 1). The results indicate that the two measurements of scab resistance (PSS and AUDPC) provide similar information and that wheat resistance to spread of scab within a spike as measured by either PSS or AUDPC is a highly heritable and stable resistance character in controlled environments.

The distributions of mean AUDPC and PSS of RILs from the 'Ning 7840'/'Clark' cross were similar in all generations evaluated and exhibited a bimodal distribution in which the numbers of lines in the two modes were in an approximate 1:1 ratio (data not shown). Therefore, a few genes with major effects can be assumed to control resistance to spread of scab in a spike in this cross.

AFLPs in wheat. DNA samples from 'Ning 7840' and 'Clark' were amplified with all 300 possible combinations of the AFLP primers described above. About $85 \%$ of the primer combinations produced clearly separated band patterns. Amplified fragments ranged in size from 50 to 900 base pairs, but most polymorphic bands ranged from 50 to 500 base pairs. Each primer combination produced 70 to 140 bands, and 2 to 18 bands per primer combination were polymorphic between the two parents. Primer combinations with three selective nucleotides amplified more bands than those primer combinations with four selective nucleotides, but in the latter case, AFLP bands were separated better. The addition of one selective nucleotide to the primer with three selective nucleotides often produced a different profile of polymorphic bands.

QTL analysis. Twenty primer combinations amplified at least one band that was polymorphic between bulked DNAs. Those primer combinations were used to associate markers and phenotypes in the $\mathrm{F}_{9}$ plants of the 133 RILs. Eleven markers showed significant association with resistance to spread of scab within a spike. Using single-marker ANOVA over four tests, the AUDPC and PSS for the two allelic classes were significantly different for 11 AFLP loci (Table 2). Allele substitution effects for both disease measurements ranged from 30 to $50 \%$ of the difference between the two parents, indicating that these markers are closely associated with major scab resistance QTL in the cross studied. Averaged over all of the evaluations, 'Ning 7840' had a PSS of about 5\% and 'Clark' averaged a PSS of $95 \%$. The range in the PSS for the population of 133 RILs was approximately 4 to $5 \%$ to $100 \%$ (data not shown).

All 11 markers showed high $R^{2}$ values, ranging from 10 to $53 \%$ in single-marker regression analysis on data from the four greenhouse

TABLE 4. Logarithm of the odds ratio and $R^{2}$ values estimated by interval mapping of 11 amplified fragment length polymorphism markers in four generations with the MAPMAKER/QTL program

\begin{tabular}{|c|c|c|c|c|c|c|c|c|}
\hline & \multicolumn{2}{|c|}{$\mathrm{F}_{5}$} & \multicolumn{2}{|c|}{$\mathrm{F}_{6}$} & \multicolumn{2}{|c|}{$\mathrm{F}_{7}$} & \multicolumn{2}{|c|}{$\mathrm{F}_{10}$} \\
\hline & $\mathrm{PSS}^{\mathrm{a}}$ & $\mathrm{AUDPC}^{\mathrm{b}}$ & PSS & AUDPC & PSS & AUDPC & PSS & AUDPC \\
\hline$\overline{R^{2}}$ & 0.32 & 0.32 & 0.30 & 0.25 & 0.56 & 0.52 & 0.54 & 0.54 \\
\hline LOD & 11.0 & 10.8 & 10.2 & 8.4 & 23.4 & 21.0 & 21.5 & 20.8 \\
\hline
\end{tabular}

a PSS $=$ Percentage of scabbed spikelets at 21 days after inoculation.

b AUDPC = Area under disease progress curve calculated from percentage of scabbed spikelets at $3,9,15$, and 21 days after inoculation. 
tests (Table 3). Except in the $\mathrm{F}_{6}$ plants, marker GCTG/CGAC1 consistently had the highest $R^{2}$ values. The maximum effect explained by the 11 markers ranged from 25 to $56 \%$ of the phenotypic variation (Table 4 ) and from 30 to $60 \%$ of the genetic variation.

Markers associated with scab resistance were all grouped in one linkage group and covered $39.4 \mathrm{cM}$ (Fig. 1). A skewed distribution with excess 'Clark'-type genotypes in respect to the expected 1:1 Mendelian ratio was observed for most of the 11 markers (Table 5). Chi-square values were significant for three of the markers. Presence of the band was associated with the resistance allele in seven markers, and with the susceptible allele in four markers. By interval analysis, one major QTL was identified between AAC/CGAC3 and GCTG/CGAC1 with LOD values from 8.4 to 23.4 (Table 4 , Fig. 1). The $F_{7}$ and $F_{10}$ plants had relatively higher $R^{2}$ and LOD values than the $\mathrm{F}_{5}$ and $\mathrm{F}_{6}$ plants. Two measurements of scab resistance in four tests were mapped to the same region of the linkage group; therefore, the putative QTL is a major QTL for scab resistance.

We attempted to map these markers to the ITMI map (14), but only one marker (GCTG/CGAC1) was polymorphic in both mapping populations. This marker was tentatively located in the long arm of chromosome 7B close to the centromere.

To explore the possibility of using these markers in marker-assisted selection, the distribution of AUDPC for a single marker (ACT/TGC7) is illustrated in Figure 2. When the marker is used as an indirect selection criterion, we would expect $32 \%$ highly resistant, 54\% moderately resistant, $14 \%$ moderately susceptible, and no highly susceptible plants to be selected.

\section{DISCUSSION}

Scab resistance assessment. To map QTL for resistance to disease, consistent disease pressure is critical for accurate assessment of the resistance potential of plant genotypes and for determination of the magnitude of the genetic factors that contribute to resistance. This is especially important for scab, since environment is one of the major determining factors for initiation and development of scab infection (4). In this study, favorable temperature and moisture conditions were provided in controlled greenhouses to minimize environmental effects on scab initiation and development. Thus, we used the controlled conditions of the greenhouse to minimize environmental variation. Since resistance to spread of scab within a spike is a major type of resistance in wheat (4), we inoculated single spikelets to assess this type of resistance. Inoculating a single spikelet in a spike eliminates differences in disease incidence among test lines and simplifies the complicated disease

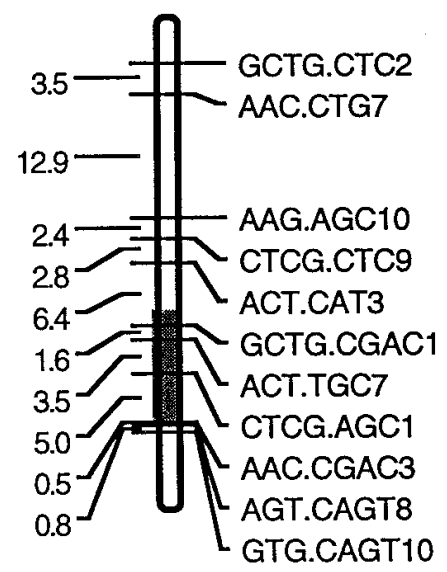

Fig. 1. Amplified fragment length polymorphism linkage map constructed from the cross 'Ning 7840 '/ Clark'. The putative scab resistance quantitative trait locus region is shown as shaded bar. Genetic distances are given on the left side of the linkage group and markers are given on the right side of the linkage group. system, since only spread of the fungus within a spike is evaluated. As a result, scab severity data from different generations and the two different assessment methods (PSS and AUDPC) were highly correlated. A high degree of consistency, or repeatability, is also indicated by the high heritability. Thus, the scab evaluation techniques in this study are suitable for QTL analysis.

Two criteria were used to describe scab severity in this study, PSS in a spike and the AUDPC. Both parameters give a measurement of spread of scab in a spike. PSS requires only one disease measurement, but the measurement must be performed at the time the inoculated plants display maximum genetic difference among genotypes. In the greenhouse conditions used in this study $\left(25^{\circ} \mathrm{C}\right)$, 3 weeks after inoculation was the appropriate time for final scab evaluation. AUDPC measures not only the final disease severity but also the progress rate in early stages of scab development. In this study, the two measurements were highly correlated and provided similar information on QTL analysis; therefore, either assessment method was adequate for differentiation of genotypes.

AFLPs in wheat. In this study, AFLPs amplified more than 100 products per primer combination and displayed polymorphisms between the two parents with almost every primer combination that amplified a clearly separated band profile. About $7 \%$ of the primer combinations amplified at least one polymorphic band between DNA bulks using AFLPs, compared with only $1 \%$ for RAPDs for the same cross (3). In the current study, 11 AFLP markers closely linked to the QTL region for scab resistance were identified. These results indicate that AFLPs are efficient for generating molecular markers for wheat scab resistance.

Since the wheat genome is relatively large, too many bands may be produced by some AFLP primer pairs with three selective nucleotides at the $3^{\prime}$ end. This may be a disadvantage of the application of AFLPs in wheat. When too many bands are produced with a primer combination, the bands are difficult to score. To solve this problem, we added one extra selective nucleotide at the $3^{\prime}$ end of AFLP primers. Primer pairs with four selective nucleotides produced fewer bands, but the bands were separated better than those produced from some primers with three selective nucleotides. The addition of an extra selective nucleotide produced different profiles of polymorphism than did the primer pairs with three selective nucleotides. For wheat AFLP analysis, combinations of primers with three and four, or four and four, selective nucleotides are recommended.

Major QTL for scab resistance. Classical genetics has demonstrated that, in controlled environments such as a greenhouse, type II scab resistance in wheat is a quantitative trait with relatively high heritability $(4,7,15)$ and controlled by few genes with major effect $(3,30)$. We identified 11 AFLP markers in one linkage group with consistently high $R^{2}$ values for scab resistance. This is the first molecular evidence of major gene control of scab resistance. $R^{2}$ values calculated from single-marker regression and

TABLE 5. $\chi^{2}$ values for 11 amplified fragment length polymorphism markers segregating in the $\mathrm{F}_{8}$ plants of the recombinant inbred line population

\begin{tabular}{lcccc}
\hline Marker $^{\mathrm{a}}$ & Band $^{\mathrm{b}}$ & 'Ning 7840' & 'Clark' & $\chi^{2}$ \\
\hline GCTG/CTC2 & $\mathrm{R}$ & 54 & 77 & $4.05^{*}$ \\
AAC/CTG7 & $\mathrm{R}$ & 53 & 73 & 3.19 \\
AAG/AGC10 & $\mathrm{R}$ & 51 & 77 & $5.30^{*}$ \\
CTCG/CTC9 & $\mathrm{S}$ & 54 & 71 & 2.33 \\
ACT/CAT3 & $\mathrm{S}$ & 62 & 59 & 0.09 \\
GCTG/CGAC1 & $\mathrm{S}$ & 57 & 74 & 2.22 \\
ACT/TGC7 & $\mathrm{R}$ & 57 & 73 & 1.98 \\
CTCG/AGC1 & $\mathrm{R}$ & 58 & 64 & 0.31 \\
AAC/CGAC3 & $\mathrm{R}$ & 53 & 70 & 2.37 \\
AGT/CAGT8 & $\mathrm{R}$ & 56 & 74 & 2.51 \\
GTG/CAGT10 & $\mathrm{S}$ & 54 & 77 & $4.05^{*}$ \\
\hline
\end{tabular}

${ }^{a}$ Marker name consists of abbreviations of selective nucleotides from the primers EcoRI (left) and MseI (right) separated by a slash.

${ }^{b} \mathrm{R}=$ presence of band was associated with low scab-severity allele, and $\mathrm{S}=$ presence of band was associated with high scab-severity allele. 
interval analysis were similar, indicating the existence of a putative major QTL. Based on the peak LOD values from interval analysis, the QTL is located between markers AAC/CGAC3 and GCTG/CGAC1 and explained up to $60 \%$ of the genetic variation. Bai (3) found that RAPD markers linked to two QTL explained $21 \%$ of the phenotypic variation. Using RFLP markers, MorenoSevilla et al. (21) identified seven chromosome regions linked to scab resistance. In their study, the RFLP markers in the seven chromosome regions explained a total of $41 \%$ of the phenotypic variation. Markers could show relatively small $R^{2}$ values because they are far from major QTL, linked to QTL with minor effects, or phenotypic variation is associated with environmental effects.

The major QTL identified in this study is very stable because it was detected in all four tests. Thus, some of the markers identified in this study with high $R^{2}$ values have good potential for use in marker-assisted selection. For example, based on the results of this study, if ACT/TGC7 is used as a selectable marker, the majority of plants (86\%) carrying the 'Ning 7840' allele for marker ACT/TGC7 will be resistant or moderately resistant. To simplify the marker-assisted selection procedure, the AFLP markers may need to be transferred to a sequence tagged site (STS) marker (or another type of marker) to improve breeding efficiency and reduce the cost of marker analysis. The identification of AFLP markers linked to a major QTL may also lay the groundwork for further map-based cloning of the major QTL; however, fine mapping on the QTL region needs to be conducted.

The unexplained variation in scab resistance could be due to QTL that remain undetected due to their minor effects or because no markers have been identified in regions associated with those QTL. The undetected QTL in the current study may result from the limitation of the bulked segregant analysis strategy, because this technique may directly target the resistance QTL with major effects, not those with minor effects (20). Small pool size (five lines of each class) in this study may also limit the power of detection of other QTL with smaller effects. Therefore, the entire wheat genome should be searched for additional resistance QTL.

Homoeologous group 7 of the wheat genome seems to play an important role in scab resistance. So far, no information on the chromosome location of scab resistance genes in 'Ning 7840' has been reported; however, by studying chromosome substitution lines, Yao et al. (33) reported that 'Sumai 3', the ancestor of 'Ning 7840', carries a major gene for resistance to spread of scab on chromosome 7A along with some others on chromosomes $2 \mathrm{~B}, 3 \mathrm{~B}$, and $6 \mathrm{~B}$. Theoretically, the major QTL in 'Ning 7840' should be the same as in 'Sumai 3'. By monosomic analysis, Yu (34) found that for seven resistant cultivars at least one chromosome in homoeolog-

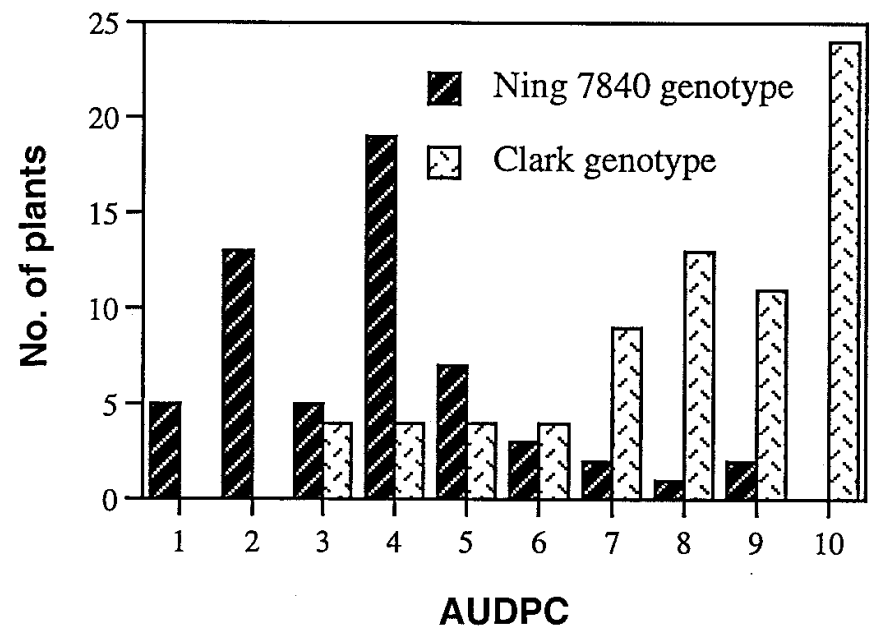

Fig. 2. Area under disease progress curve (AUDPC) (calculated from percentage of scabbed spikelets at 3,9, 15, and 21 days after inoculation) distribution of two allelic classes of marker ACT/TGC7. ous group 7 carried genes for resistance to scab. Assuming that the same AFLP bands segregating in different mapping populations are mainly shared alleles $(11,24)$, the major QTL identified in this study was tentatively mapped in chromosome $7 \mathrm{~B}$ using comparative mapping with AFLP markers on the two mapping populations. One shared marker between the two populations may not be sufficient to map the QTL to a chromosome. Therefore, to confirm the chromosome location of the QTL, comparative mapping of the two populations should be conducted using additional AFLPs or other types of molecular markers.

\section{ACKNOWLEDGMENTS}

Financial support for this research was partially provided by the Illinois Council for Food and Agricultural Research. We thank S. Goodwin and X.-Y. Hu (Department of Botany and Plant Pathology, Purdue University) for providing DNA of the ITMI mapping population.

\section{LITERATURE CITED}

1. Alonso-Blanco, C., Peters, A. J. M., Koornneef, M., Lister, C., Dean, C., ven den Bosch, N., Pot, J., and Kuiper, M. T. R. 1998. Development of an AFLP based linkage map of Ler, $\mathrm{Col}$ and Cvi Arabidopsis thaliana ecotypes and construction of a Ler/Cvi recombinant inbred line population. Plant J. 14:259-271.

2. Autrique, E., Singh, R. P., Tanksley, S. D., and Sorrells, M. E. 1995. Molecular markers for four leaf rust resistance genes introgressed into wheat from wild relatives. Genome 38:75-83.

3. Bai, G.-H. 1995. Scab of wheat: Epidemiology, inheritance of resistance and molecular markers linked to cultivar resistance. Ph.D. thesis. Purdue University, West Lafayette, IN.

4. Bai, G.-H., and Shaner, G. 1994. Scab of wheat: Prospects for control. Plant Dis. 78:760-766.

5. Bai, G.-H., and Shaner, G. 1996. Variation in Fusarium graminearum and cultivar resistance to wheat scab. Plant Dis. 80:975-979.

6. Chao, S., Sharp, P. J., Worland, A. J., Warham, E. J., Koebner, R. M. D., and Gale, M. D. 1989. RFLP-based genetic linkage maps of wheat homoeologous group 7 chromosomes. Theor. Appl. Genet. 78:495-504.

7. Chen, C.-H. 1983. A study on the inheritance of scab-resistance in wheat. Acta Agric. Univ. Zhejiang 9:115-126.

8. Cnops, G., den Boer, B., Gerats, A., Van Montagu, M., and Van Lijsebettens, M. 1996. Chromosome landing at Arabidopsis TORNADO1 locus using AFLP-based strategy. Mol. Gen. Genet. 253:32-41.

9. El Attari, H., Rebai, A., Hayes, P. M., Barrault, G., Dechamp-Guillaume, G., and Sarrafi, A. 1998. Potential of double-haploid line and localization of quantitative trait loci (QTL) for partial resistance to bacterial leaf streak (Xanthomonas campestris pv. hordei) in barley. Theor. Appl. Genet. 96:95-100.

10. Horvath, D. P., Dahleen, L. S., Stebbing, J. A., and Penner, G. 1995. A codominant PCR-based marker for assisted selection of durable stem rust resistance in barley. Crop Sci. 35:1445-1450.

11. Jin, H., Domier, L. L., Kolb, F. L., and Brown, C. M. 1998. Identification of quantitative loci for tolerance to barley yellow dwarf virus in oat. Phytopathology 88:410-415.

12. Kosambi, D. D. 1944. The estimation of map distances from recombination values. Ann. Eugen. 12:172-175.

13. Lander, E., Green, P., Abrahamson, J., Barlow, A., Daley, M., Lincoln, S., and Newburg, L. 1987. MAPMAKER: An interactive computer package for constructing primary genetic linkage maps of experimental and natural populations. Genomics 1:171-181.

14. Leroy, P., Negre, S., Tixier, M. H., Perretant, M. R., Sourdille, P., Gay, G., Bernard, M., Coville, J. L., Quetier, F., Nelson, C., Sorrells, M., Marino, C. L., Hart, G., Friebe, B., Gill, B. S., and Roder, M. 1997. A genetic reference map for the bread wheat genome, Triticum aestivum $\mathrm{L}$ em. Thell. Pages 134-140 in: Progress in Genome Mapping of Wheat and Related Species. Joint Proc. Public Workshops Int. Triticeae Mapping Initiative, 5th and 6th. P. E. McGuire and C. O. Qualset, eds. Genetic Resources Conservation Program, Division of Agricultural and Natural Resources, University of California, Davis.

15. Li, Y.-F., and Yu, Y.-J. 1988. A diallel analysis on resistance index to scab in seven wheat cultivars. J. Huazhong Agric. Univ. 7:7-14.

16. Ma, Z.-Q., Sorrells, M. E., and Tanksley, S. D. 1994. RFLP markers linked to powdery mildew resistance genes Pm1, Pm2, Pm3 and Pm4 in wheat. Genome 37:871-885.

17. Maughan, P. J., Saghai-Maroof, M. A., and Buss, G. R. 1996. Molecularmarker analysis of seed-weight: Genomic locations, gene action, and evidence for orthologous evolution among three legume species. Theor. 
Appl. Genet. 93:574-579.

18. Mesterhazy, A. 1995. Types and components of resistance against Fusarium head blight of wheat. Plant Breed. 114:377-386.

19. Michelmore, R. W. 1995. Isolation of disease resistance genes from crop plants. Curr. Opin. Biol. 6:145-152.

20. Michelmore, R. W., Paran, I., and Kesseli, R. V. 1991. Identification of markers linked to disease resistance genes by bulked segregant analysis: A rapid method to detect markers in specific genomic regions by using segregating populations. Proc. Natl. Acad. Sci. U.S.A. 88:9828-9832.

21. Moreno-Sevilla, B., Anderson, J. A., Waldron, B. L., Stack, R. W., and Frohberg, R. C. 1997. RFLP mapping of Fusarium head blight resistant genes in wheat. Page 72 in: Proc. Natl. Fusarium Head Blight Forum. St. Paul, MN.

22. Ohm, H. W., Shaner, G., Foster, J. E., Patterson, F. L., and Buechley, G. 1988. Registration of 'Clark' wheat. Crop Sci. 28:1032.

23. Powell, W., Morgante, M., Andre, C., Hanafey, M., Vogel, M. J., Tingey, S. V., and Rafalski, A. 1996. The comparison of RFLP, RAPD, AFLP and SSR (microsatellite) markers for germplasm analysis. Mol. Breed. 2:225-238.

24. Rouppe van der Voort, J. N. A. M., van Zandvoort, P., van Eck, H. J., Folkertsma, R. T., Hutten, R. C. B., Graaistra, J., Gommers, F. J., Jacobsen, E., Helder, J., and Bakker, J. 1997. Use of allele specificity of comigrating AFLP markers to align genetic maps from different potato genotypes. Mol. Gen. Genet. 255:438-447.

25. Saghai-Maroof, M. A., Soliman, K. M., Jorgensen, R. A., and Allard, R. W. 1984. Ribosomal DNA spacer-length polymorphisms in barley: Mendelian inheritance, chromosomal location, and population dynamics. Proc. Natl. Acad. Sci. U.S.A. 81:8014-8018.

26. Saghai-Maroof, M. A., Yue, Y. G., Xiang, Z. X., Stromberg, E. L., and
Rufener, G. K. 1996. Identification of quantitative trait loci controlling resistance to gray leaf spot disease in maize. Theor. Appl. Genet. 93:539-546.

27. Shaner, G., and Finney, R. E. 1977. The effect of nitrogen fertilization on the expression of slow-mildewing resistance in Knox wheat. Phytopathology 67:1051-1056.

28. Steel, R. G. D., and Torrie, J. H. 1980. Principles and Procedures of Statistics: A Biometrical Approach. 2nd ed. McGraw-Hill Publ., Inc., New York.

29. Thomas, C. M., Vos, P., Zabeau, M., Jones, D. A., Norcott, K. A., Chadwick, B. P., and Jones, J. D. G. 1995. Identification of amplified restriction fragment polymorphism (AFLP) markers tightly linked to the tomato cf-9 gene for resistance to Cladosporium fulvum. Plant J. 8:785-794.

30. Van Ginkel, M., Van der Schaar, W., Zhuping, Y., and Rajaram, S. 1996. Inheritance of resistance to scab in two wheat cultivars from Brazil and China. Plant Dis. 80:863-967.

31. Vos, P., Hogers, R., Bleeker, M., Reijans, M., van de Lee, T., Hornes, M., Frijters, A., Pot, J., Peleman, J., Kuiper, M., and Zabeau, M. 1995. AFLP: A new technique for DNA fingerprinting. Nucleic Acids Res. 23: 4407-4414.

32. Williams, K. J., Fisher, J. M., and Langridge, P. 1994. Identification of RFLP markers linked to the cereal cyst nematode resistance gene (Cre) in wheat. Theor. Appl. Genet. 89:927-930.

33. Yao, J.-B., Ge, Y.-F., Wang, S.-W., Yao, G.-C., Zhou, C.-F., and Qian, C.-M. 1995. Chromosomal location of genes for scab resistance in wheat cultivar Sumai 3. Acta Agron. Sin. 23:450-453.

34. Yu, Y.-J. 1990. Genetic analysis for scab resistance in four wheat cultivars, PHJZM, HHDTB, CYHM and YGFZ. Pages 197-205 in: Advance in Research on Inheritance of Resistance to Diseases in Major Crops. L.-H. Zhu, W.-Z. Lu, and Y.-F. Xie, eds. Jiangsu Sci.-Tech. Publ., Nanjing, China. 